\title{
A comparison of computational and experimental lift-off heights of coflow laminar diffusion flames
}

\author{
Kevin T. Walsh ${ }^{\mathrm{a}}$, Joseph Fielding ${ }^{\mathrm{a}}$, Mitchell D. Smooke ${ }^{\mathrm{a}, *}$, \\ Marshall B. Long ${ }^{a}$, Amable Liñán ${ }^{b}$ \\ ${ }^{a}$ Department of Mechanical Engineering, Yale University, New Haven, CT 06520-8284, USA \\ ${ }^{\mathrm{b}}$ Department of Motorpropulsion y Termofluidodinamica, Universidad Politécnica de Madrid, 28040 Madrid, Spain
}

\section{Introduction}

The ability to predict the coupled effects of complex transport phenomena with detailed chemical kinetics in diffusion flames is critical in the modeling of turbulent reacting flows and in understanding soot formation and radiative transfer. In addition, the factors that affect extinction

\footnotetext{
* Corresponding author. Fax: +1 2034326775 .

E-mail address: mitchell.smooke@yale.edu (M.D. Smooke).
}

in diffusion flames are of practical importance in areas such as fire suppression and engine efficiency. The goal of our characterizations of coflow laminar diffusion flames was to provide a broader understanding of the successes and limitations of current combustion models. This will lead to a more detailed understanding of the interaction of convection, diffusion, and chemistry in both buoyant and non-buoyant environments.

In this paper, we study lifted axisymmetric laminar diffusion flames, which have been previously characterized both experimentally and 
computationally [1-5]. The fuel, nitrogen-diluted methane, is surrounded by an air coflow. Experimentally, temperature and major species concentrations were simultaneously measured with Rayleigh and Raman scattering [4]. Laser-induced fluorescence (LIF) measurements were performed to measure number densities of minor species. Quantitative, linear LIF measurements were made for $\mathrm{OH}$ [2], $\mathrm{NO}$ [3], and $\mathrm{CH}$ [5]. Modeling work has employed several kinetic schemes, including a 26 species $\mathrm{C}_{2}$ hydrocarbon mechanism [2] and GRIMech 2.11 [6]. Both produced excellent agreement for temperature and major species [4]. Computed peak concentrations of $\mathrm{OH}$ and $\mathrm{CH}$ were within $15 \%$ and $20 \%$, respectively, of their measured values.

Previous laboratory studies performed on this flame indicated that quantitative number densities of excited-state $\mathrm{CH}\left(\mathrm{A}^{2} \Delta\right.$, denoted $\left.\mathrm{CH}^{*}\right)$, and excited-state $\mathrm{OH}\left(\mathrm{A}^{2} \Sigma\right.$, denoted $\left.\mathrm{OH}^{*}\right)$ could be measured in a short time with a simple, compact optical setup that could be made compatible with existing reduced gravity facilities [5]. Although reactions involving $\mathrm{CH}^{*}$ and $\mathrm{OH}^{*}$ are not included in most kinetics models, the $\mathrm{CH}^{*}, \mathrm{OH}^{*}$, and ground-state $\mathrm{CH}$ distributions are spatially coincident in the flame anchoring region [5]. Therefore, the ground-state $\mathrm{CH}$ distribution and the readily measured $\mathrm{CH}^{*} / \mathrm{OH}^{*}$ distributions can be used to provide a consistent and convenient way of comparing lift-off height and flame shape in the diffusion flames under investigation. Given that the fuel composition affects flame chemistry and that buoyancy influences the velocity profile of the flow, we have the opportunity to study the chemistry and fluid dynamic interaction computationally and experimentally. In performing this normal and reduced gravity study, calculations were made for a range of gravity and flow conditions. Furthermore, modifications to the experimental approach were required as a consequence of the constraints imposed by the reduced gravity environment of the $\mathrm{KC}-135$. Results from the experiments and computations are presented in the following sections.

\section{Burner configuration}

The burner used in this experiment contains a central fuel jet ( $4 \mathrm{~mm}$ inner diameter, $0.4 \mathrm{~mm}$ wall thickness) surrounded by coflowing air $(50 \mathrm{~mm}$ diameter). The standard flow conditions previously studied in normal gravity consist of fuel composed of $65 \% \mathrm{CH}_{4}$ diluted with $35 \% \mathrm{~N}_{2}$ by volume (denoted $65 / 35$ in later discussion). The plug flow exit velocity of both fuel and coflow was $35 \mathrm{~cm} / \mathrm{s}$. These conditions produce a blue flame roughly $3 \mathrm{~cm}$ in length with a lift-off height of $5.5 \mathrm{~mm}$ in normal gravity. A wide range of flow conditions were measured in this study, with the $\mathrm{CH}_{4} / \mathrm{N}_{2}$ fuel composition varying from $100 \%$ $\mathrm{CH}_{4}$ (denoted 100/0) to $40 \% \mathrm{CH}_{4}$ (denoted 40 /
60 ) in $5 \%$ increments, with fuel and air exit velocities held fixed at $35 \mathrm{~cm} / \mathrm{s}$.

\section{Experimental setup}

Reduced gravity facilities impose restrictions on experiment size, power consumption, and complexity. To be consistent with these requirements and to still provide quantitative information that can be compared with models, the approach in this work is to perform emission tomography on selected chemiluminescent flame radicals. These reconstructed emission measurements result in an intensity distribution proportional to number density. This intensity level can be calibrated, post-flight, with Rayleigh scattering [7], which was done on a flow of clean air with the same optical setup as the emission measurements. A quantitative, spatially resolved number density profile can then be obtained through tomographic inversion and a spectral throughput correction.

Several modifications to the laboratory-based experimental configuration were required to make flame emission measurements aboard the KC-135 reduced-gravity aircraft. The burner and ignition system were housed inside a pressure vessel to maintain standard atmospheric pressure. This was done for flame stability as well as to mimic laboratory and computed conditions. The vessel contained a quartz window to provide optical access, as well as two other ports covered by aluminum plugs that could be removed in a non-flight situation to perform laser calibration. The spectrally filtered chemiluminescence images are acquired by a cooled, unintensified CCD detector and provide quantitative intensity information. A color video camera is used to give qualitative insight into flame structure and soot production.

Pressure at the vent of the pressure vessel ranges from one atmosphere to $\sim 0.25 \mathrm{~atm}$ (aircraft at maximum altitude). To keep the combustion vessel at standard atmospheric pressure for all these conditions, an orifice plate and a computer-controlled servo valve are utilized in parallel. This exhaust system kept the combustion vessel pressure at standard atmospheric pressure to better than $1 \%$ for the duration of the flight.

During each low-gravity maneuver, the combustion vessel pressure and airplane accelerometer signals were simultaneously recorded with the flame emission signal. Flame emission images analyzed in this study typically had a gravitational time-trace average of $0.01 g_{\mathrm{EARTH}}$, with rms fluctuations of $0.02 g_{\text {EARTH }}$.

\section{4. $\mathrm{OH}^{*}$ and $\mathrm{CH}^{*}$ measurements}

The $\mathrm{A}^{2} \Sigma \rightarrow \mathrm{X}^{2} \Pi \quad \mathrm{OH}^{*}$ chemiluminescence band peaks at $307.8 \mathrm{~nm}$. Excited-state $\mathrm{OH}$ 
measurements were made with a cooled $\mathrm{CCD}$ camera using a $f / 4.5$ quartz camera lens; the camera/lens system was placed $50 \mathrm{~cm}$ away to ensure a wide depth of field. A narrow bandpass interference filter (center $307 \mathrm{~nm}, 10 \mathrm{~nm}$ bandwidth) was used to reject other wavelengths. High emission signal levels were collected with $10 \mathrm{~s}$ integration times. Post-flight Rayleigh calibration was done with a Nd:YAG-pumped dye laser utilizing sulfarhodamine 640 dye. The $612 \mathrm{~nm}$ output was frequency doubled to perform calibration measurements on clean air. Beam energy in the ultraviolet was varied from 0.3 to $2.0 \mathrm{~mJ} / \mathrm{pulse}$, with the signal collected over 600 shots.

For $\mathrm{CH}^{*}$ flame emission, the $\mathrm{A}^{2} \Delta \rightarrow \mathrm{X}^{2} \Pi$ transition at $431.2 \mathrm{~nm}$ was imaged with the camera and lens system detailed above. An interference filter (center $431 \mathrm{~nm}, 10 \mathrm{~nm}$ bandwidth) was used to isolate the $\mathrm{CH}^{*}$ emission. The post-flight calibration measurements were similarly performed with a Nd:YAG-pumped dye laser. During Rayleigh calibration, the energy of the $431.5 \mathrm{~nm}$ beam was varied between 0.5 and $2.5 \mathrm{~mJ}$, with an integration of 600 shots.

The emission signal is given by [8]:

$S_{\mathrm{em}}=\frac{1}{4 \pi} A_{21} \tau V_{\mathrm{em}} N^{*} \Omega \varepsilon \eta$,

where $A_{21}$ is the Einstein $A$ coefficient, $\tau$ is the integration time, $V_{\mathrm{em}}$ is the emission pixel volume, $N^{*}$ is the number density of excited-state molecules, $\Omega$ is the solid angle, $\varepsilon$ is a calibration constant of the optics, and $\eta$ is the detector efficiency in counts per photon. Since the chemically excited radicals being measured are in steady-state, no calculation of collisional quenching is required [8], in contrast to the analysis reported in earlier work [5]. A detailed discussion of extracting number density from chemiluminescence measurements and the kinetic modeling of the $\mathrm{CH}^{*}$ and $\mathrm{OH}^{*}$ radicals has been published [9].

To relate the measured flame chemiluminescence signal to a quantitative concentration, the relationship between detected counts and photons emitted must be known. Luque and Crosley [7] have demonstrated that Rayleigh scattering can be used to calibrate the detection system and solve for the product $(\Omega \varepsilon \eta)$ in Eq. (1). The chemiluminescence detection apparatus is used to measure laser Rayleigh scattering from a gas of known temperature, pressure, and composition. [7]

The Rayleigh signal from a single laser pulse is

$S_{\mathrm{R}}=\frac{N E_{\mathrm{R}} V_{\mathrm{R}}\left(\frac{\partial \sigma}{\partial \Omega}\right)}{A_{\mathrm{R}} h v} \Omega \varepsilon \eta$,

where $N$ is the known number density of the gas, $E_{\mathrm{R}}$ is the laser energy, $V_{\mathrm{R}}$ is the volume over which the Rayleigh signal is collected, $\partial \sigma / \partial \Omega$ is the Rayleigh cross-section, $A_{\mathrm{R}}$ is the cross-sec- tional area of the laser beam, and $h v$ is the laser photon energy. Although calibration can be done with a single Rayleigh image, measurements were made over a range of energies to verify the linear relationship between the signal and laser energy, and to confirm a zero intercept. Additionally, the spectral distributions of the Rayleigh signal and chemiluminescence signal differ, giving the two signals distinct spectral overlaps with the interference filter used in the detection system. $\Gamma$ is defined as the ratio of these two spectral overlaps and can be explicitly written as

$\Gamma=\frac{\int_{0}^{\infty} E(\lambda) F(\lambda) \mathrm{d} \lambda}{\int_{0}^{\infty} R(\lambda) F(\lambda) \mathrm{d} \lambda}$,

where $F(\lambda)$ is the interference filter throughput, $E(\lambda)$ is the emission profile, and $R(\lambda)$ the Rayleigh profile, with the integrations taking place over all wavelengths $\lambda . E(\lambda)$ and $R(\lambda)$ are normalized such that:

$\int_{0}^{\infty} E(\lambda) \mathrm{d} \lambda=\int_{0}^{\infty} R(\lambda) \mathrm{d} \lambda=1$.

Eqs. (1) and (2) can now be combined to yield

$N^{*}=\frac{4 \pi \Gamma S_{\mathrm{em}} N E_{\mathrm{R}} V_{\mathrm{R}}\left(\frac{\partial \sigma}{\partial \Omega}\right)}{S_{\mathrm{R}} A_{21} \tau V_{\mathrm{em}} A_{\mathrm{R}} h v}$.

\section{Image processing and corrections}

Appropriate background images, taken for both $\mathrm{CH}^{*}$ and $\mathrm{OH}^{*}$ with the flame extinguished, are subtracted from the raw emission signal. Normally, quantitative imaging techniques also require correction for variations in the throughput of the imaging optics. However, due to the large object distance and depth-of-field, the variation in throughput was less than $2 \%$ across the image (determined from the laser calibration images), and no response correction was included. Since the measured emission signals are integrated through the collection optics along the line of sight, a tomographic inversion procedure is required to reconstruct the planar, spatially resolved intensity distribution. When thin, parallel rays are collected from an unknown axisymmetric distribution, an Abel inversion can be used to reconstruct the distribution from a measured intensity profile [10]. However, a single lens collects nonparallel light rays in a cone, over a non-zero solid angle. A ray tracing algorithm [11] confirmed that the predicted signal profile matched measurement, and the inverted radial distribution did not spatially differ from that which would result from collecting precisely parallel rays. Further confirmation that spatial distortions were insignificant came from previous work, in which the groundstate $\mathrm{CH}$ profile and the Abel-inverted $\mathrm{CH}^{*}$ distribution were measured to be spatially coincident 
[5], even though a less favorable optical configuration was used. Therefore, given the flame's axisymmetry and the chosen optical configuration, we can recover a two-dimensional, in-plane intensity distribution proportional to number density with the use of an algorithm that is equivalent to a two-point Abel deconvolution [10]. After inversion, pixel volumes were determined to be cubes of side length $68 \mu \mathrm{m}$ for both $\mathrm{OH}^{*}$ and $\mathrm{CH}^{*}$. The spatial resolution is estimated at $140 \mu \mathrm{m}$ (about 2 pixel widths), which is adequate for resolving peak concentrations, as well as determining flame shape and lift-off height.

A value for $\Gamma$ must be computed to correct for the throughput differences of the emission and calibration signals. The Rayleigh signal has a spectral width equal to the laser linewidth of $0.03 \AA$, and therefore passes through the interference filter at the laser wavelength only. In contrast, the chemiluminescence signals are spectrally broad, making the amount of light collected dependent on the entire spectral overlap with the interference filter in the optical setup. LIFBASE [12] was used to calculate the $\mathrm{CH}^{*}$ and $\mathrm{OH}^{*}$ spectral emission profiles $E(\lambda)$ and a spectrophotometer was used to measure the interference filter transmission, $F(\lambda)$. The resulting correction factor, $\Gamma$, is 0.78 for $\mathrm{CH}^{*}$ and 0.99 for $\mathrm{OH}^{*}$.

The spatial location of the computed $\mathrm{CH}$ profile was used to define the flame sheet region where $\mathrm{CH}^{*}$ and $\mathrm{OH}^{*}$ coexist. The computations indicated that both of these radicals are present in a hot, thin zone at a constant temperature that varies from $1877 \mathrm{~K}$ at $50 / 50$ to $1940 \mathrm{~K}$ at $75 / 25$, independent of buoyancy. The absolute number densities that result from corrected measurements are combined with computed temperatures to provide $\mathrm{CH}^{*}$ and $\mathrm{OH}^{*}$ mole fractions. The peak mole fractions of $\mathrm{CH}^{*}$ varied between $0.8 \times 10^{-11}$ and $1.4 \times 10^{-11}$, and the corresponding peaks of $\mathrm{OH}^{*}$ varied between $3.2 \times 10^{-11}$ and $4.3 \times 10^{-11}$ as the fuel composition was varied from $50 / 50$ to $75 /$ 25. Uncertainty in these measurements comes from the Rayleigh calibration and the flame emission signal. For a perfectly axisymmetric flame, the integrated flame emission signal would be symmetric about the jet centerline. In our measurements, slight differences existed between the two halves of the measured signal. The developers of the Rayleigh calibration procedure used here assessed its relative error to be $10-15 \%$ [7]. These factors combine to produce a measured mole fraction accuracy of $25 \%$ for $\mathrm{CH}^{*}$ and $\mathrm{OH}^{*}$, which is comparable to that of previous measurements of trace species in this flame $[3,5]$.

\section{Computational approach}

The computational model solves the full set of elliptic two-dimensional governing equations for mass, momentum, species, and energy conservation on a two-dimensional mesh using a velocity-vorticity formulation [3]. The resulting non-linear equations are then solved on an IBM RS/6000 Model 590 computer by a combination of time integration and Newton's method. Flame structure was calculated over a range of flow conditions in both zero gravity and normal gravity. The results of a computed solution at standard flow conditions $(65 / 35)$ and normal gravity were used as a starting point. In subsequent calculations, the value of the gravitational acceleration (g) was reduced by $10 \mathrm{~cm} / \mathrm{s}^{2}$, and a new solution was calculated using Newton's method. A range of mixture conditions were reached by using the $65 / 35$ flame as an initial condition and varying the fuel mixture in $5 \%$ increments.

All calculations performed in this study used the 26-species $C_{2}$ mechanism [2], which produces better agreement with measured lift-off height than GRI-Mech 2.11 at the 65/35 flow condition. Computations performed at this flow condition with different values for the gravitational constant indicated that gravity plays an important role in both the size and shape of the coflow laminar diffusion flame [13]. Computations were performed for $\mathrm{CH}_{4} / \mathrm{N}_{2}$ mixtures ranging from $30 / 70$ to $75 / 25$.

\section{Results and discussion}

Measurements of the airplane accelerometer quantified the positive and negative unsteady forces $(g$-jitter) present during a given parabola. Understanding the behavior of the flame in $g$-jitter is crucial to interpreting measurements on the $\mathrm{KC}-135$. Our measurements require a stable emission signal over the duration of a $10 \mathrm{~s}$ camera exposure. $\mathrm{CH}^{*}$ and $\mathrm{OH}^{*}$ concentrations peak strongly in the flame anchoring region and fall off rapidly along the flame length, as seen at the 65/35 flow condition in normal gravity in Fig. 1. During any given reduced gravity maneuver, the flame base appears extremely stable on the video monitor, which allowed for careful emission measurements of both $\mathrm{CH}^{*}$ and $\mathrm{OH}^{*}$ for fuel compositions ranging from $50 / 50$ to $100 / 0$. The flame tip, however, can behave differently than the flame anchoring region. In dilute fuel blends $(40-50 \%$ $\mathrm{N}_{2}$ ), the entire reduced gravity flame appeared as stable as a normal gravity flame. For richer fuel mixtures, containing more than $75 \% \mathrm{CH}_{4}$ by volume, the reduced gravity flames produced significant soot luminescence, which could be seen to fluctuate as a result of $g$-jitter. In the 100/0 flame, $g$-jitter causes the sooty region in the flame to "bounce," translate, and change shape considerably.

Measurements were performed in reduced gravity and normal gravity over a wide range of flow conditions. Measured flame shape, as 

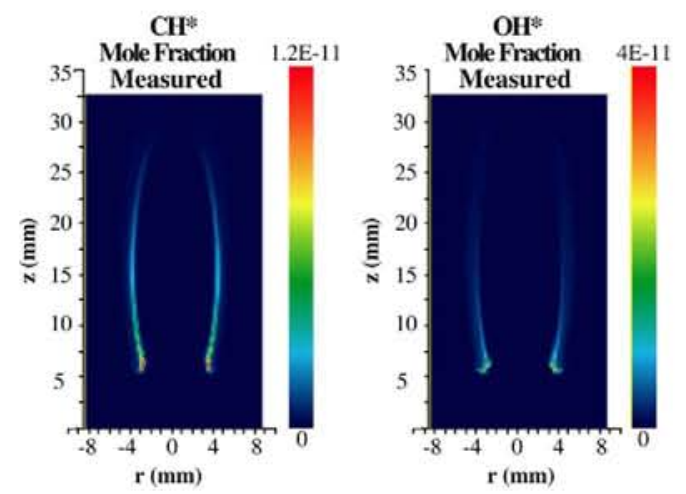

Fig. 1. Measured $\mathrm{CH}^{*}$ and $\mathrm{OH}^{*}$ distributions at $65 / 35$, normal gravity.

indicated by the spatial distributions of the $\mathrm{CH}^{*}$ and $\mathrm{OH}^{*}$ radicals, can significantly change between normal gravity and reduced gravity. In general, a reduced gravity flame is shorter, wider, and has a higher flame front curvature relative to its normal gravity counterpart. Also, the normal gravity flame has a higher lift-off height than the corresponding reduced gravity flame. These effects can be seen in the measured $1 \mathrm{~g}$ and low-g $\mathrm{CH}^{*}$ profiles shown in Fig. 2. Of the four flames, the difference in lift-off height is greatest for the 50/ 50 fuel mixture. Note for the $80 / 20$ and $100 / 0$ flow conditions at reduced gravity, soot luminescence is visible at the flame tip within the $\mathrm{CH}^{*}$ detection bandpass. Computations were not performed for these sooty flames. However, flame sheet temperatures were linearly extrapolated to these flow conditions to provide a peak mole fraction for visualization purposes. The $\mathrm{OH}^{*}$ profile is more localized than that of $\mathrm{CH}^{*}$, although similar gravity-induced changes in flame structure can be seen. Soot luminescence is not visible within the bandpass of the $\mathrm{OH}^{*}$ filter.

Computed $\mathrm{CH}$ profiles are shown with measured $\mathrm{CH}^{*}$ profiles in Fig. 3. At standard flow conditions $(65 / 35)$, the structural agreement is excellent for both lift-off height and flame shape, both in normal and reduced gravity. Although the computed flame length may appear to be over-predicted at this flow condition, $\mathrm{CH}^{*}$ does not exist at the flame tip and as such is not a good indicator of flame length.

We define the measured lift-off height as the height above the burner where the $\mathrm{CH}^{*} / \mathrm{OH}^{*}$ maximum occurs, and similarly for $\mathrm{CH}$ in the computations. All three peaks occupy the same spatial location, as shown in earlier work [5]. The measured and computed lift-off heights, both in
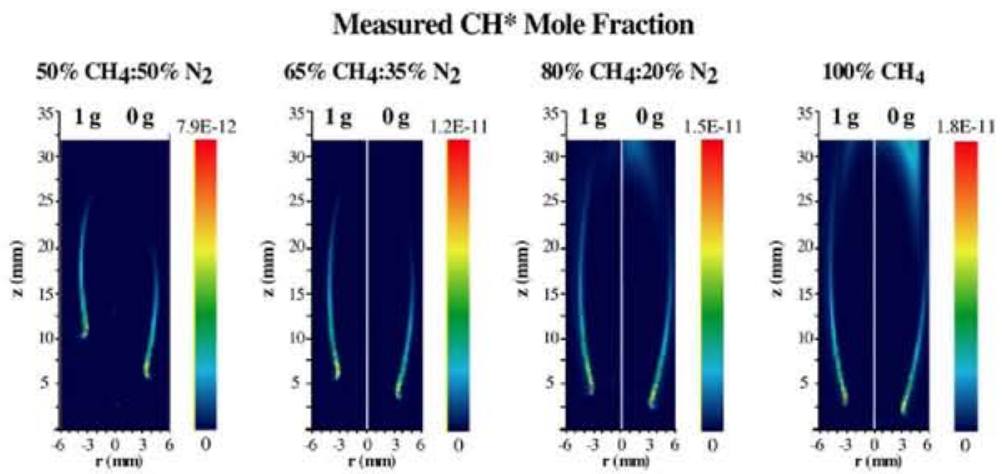

Fig. 2. Measured $\mathrm{CH}^{*}$ profiles in normal and reduced gravity over a range of fuel mixtures.
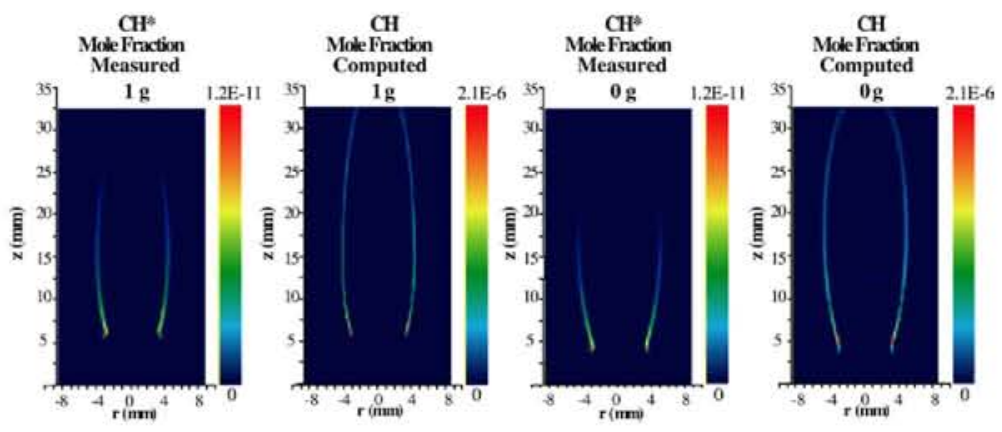

Fig. 3. Measured $\mathrm{CH}^{*}$ and computed (26-species $\mathrm{C}_{2}$ mechanism) $\mathrm{CH}$ profiles in normal and reduced gravity at $65 / 35$. 
reduced gravity and normal gravity, can now be plotted as a function of methane level in the fuel stream as shown in Fig. 4. In addition to the lift-off heights derived from the emission measurements discussed here, Fig. 4 shows lift-off heights for greater dilution levels obtained from temperature measurements in the same flame, described in [13]. Uncertainties in measured lift-off heights at higher dilution levels arise from flame asymmetries and are likely responsible for the discrepancies between the two measurement techniques.

The predicted lift-off height agrees well with measurement in $1 g$ at the $75 / 25$ and $65 / 35$ fuel mixtures. As the methane level decreases from $65 \%$ to $50 \%$, the computed and measured curves begin to separate. When the fuel mixture is diluted below $50 \% \mathrm{CH}_{4}$ in $1 \mathrm{~g}$, the lift-off height becomes increasingly under-predicted, until the code computes a stable flame at fuel mixtures $(35 \%$ and $30 \% \mathrm{CH}_{4}$ ) beyond the $1 \mathrm{~g}$ experimental blow-off limit $\left(40 \% \mathrm{CH}_{4}\right)$. Further, the difference between computed normal and reduced gravity lift-off heights does not match the measured curves, which separate increasingly as the fuel mixture is diluted. The measured and computed reduced gravity lift-off heights show reasonable agreement up to a dilution level of $50 \%$ and then depart significantly.

A number of computational, fluid dynamic, and transport modifications were made to the model to try to understand the lift-off discrepancies. Specifically, in the neighborhood of the flame front, the grid resolution was increased by a factor of 4 . We also relaxed the downstream (zero axial gradient) and far-field (zero radial gradient) boundary conditions. In addition, the diffusion coefficients of the fuel and hydrogen were increased by $10 \%$, and we tightened (factor of 100 ) the computational tolerances. Changes to these

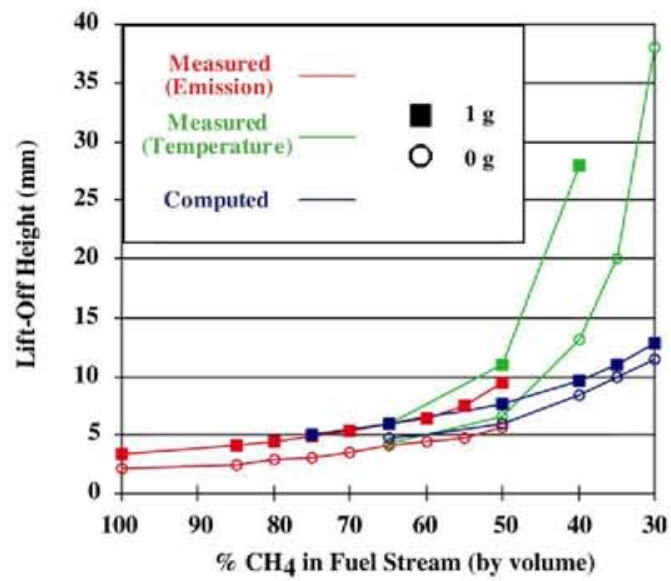

Fig. 4. Measured and computed (26-species $\mathrm{C}_{2}$ mechanism) lift-off heights in normal and reduced gravity. parameters were seen to have only minimal impact on predicted lift-off heights, indicating that some other process is controlling the discrepancies at high dilution levels. To aid in the analysis of this problem, it is worthwhile to interpret the lift-off results using (see also [14-22]) the structure of edge flames and triple flame propagation along mixing layers.

The lift-off height in diffusion flames is determined by the need to balance convective heat transport due to the upstream flow with upstream heat conduction from the reaction zone, which lies close to the stoichiometric surface. Specifically, upstream of the flame edge, mixing of the fuel and air takes place in a mixing layer, across which the mixture runs from very lean to very rich. At small heights $z$ from the injector, under reduced gravity conditions, the thickness, $\delta_{\mathrm{m}}$, of the mixing layer grows like $\left(D_{\mathrm{F}} z / U\right)^{1 / 2}$ where $D_{\mathrm{F}}$ is the fuel diffusivity, and $U$ is the equal flow velocity of the two streams, which does not grow with $z$. The growth of $\delta_{\mathrm{m}}$ with $z$ will be weaker under normal gravity, $g$, when the vertical velocity $u_{\mathrm{M}}$ of the methane stream grows with $z$ as $\left(u_{\mathrm{M}}\right)^{2}=U^{2}+2 g z\left(1-\rho_{\mathrm{M}} / \rho_{\mathrm{A}}\right)$. This growth of $u_{\mathrm{M}}$ is due to the buoyancy forces in the methane stream, of lower density, $\rho_{\mathbf{M}}$, than the density, $\rho_{\mathrm{A}}$, of the outer air stream.

The edge of the flame lies close to the stoichiometric surface, where the effective reaction time is minimum due to the higher flame temperatures, and its position is determined by the necessary balance among reaction, upstream diffusion, and convection, with a velocity between $u_{\mathrm{M}}$ and $U$. For moderately large lift-off heights, the thickness of the mixing layer, just upstream of the lift-off height, is large compared with the thickness $\delta_{\mathrm{L}}=D_{\mathrm{F}} / S_{\mathrm{L}}$ of the planar stoichiometric flame of speed $S_{\mathrm{L}}$ at the flame front. The lift-off conditions should in this case result by equating $S_{\mathrm{L}}$ to the upstream flow velocity, which is $U$ under reduced gravity conditions, and larger under normal gravity. However, due to thermal expansion effects, the triple flame propagation velocity $U_{\mathrm{F}}$ is higher than $S_{\mathrm{L}}$ for methane air flames and approaches a constant value for large enough values of the Damköhler number

$D_{a}=\left(\delta_{\mathrm{m}} / \delta_{\mathrm{L}}\right)^{2}=\left(S_{\mathrm{L}} \delta_{\mathrm{m}} / D_{\mathrm{F}}\right)^{2}$.

With decreasing values of the Damköhler number, the flame front structure changes, due to curvature effects, from a triple flame to an edge flame, where the premixed flame appears to merge with the diffusion flame. In addition, the ratio $U_{\mathrm{F}} /$ $S_{\mathrm{L}}$ is a function of the Damköhler number

$U_{\mathrm{F}} / S_{\mathrm{L}}=F\left(S_{\mathrm{L}}^{2} \delta_{\mathrm{m}}^{2} / D_{\mathrm{F}}^{2}\right)$

that decreases when the Damköhler number decreases. The transition in the front structure and the lowering of $U_{\mathrm{F}} / S_{\mathrm{L}}$ occur at values of $D_{a}$, of 
order 100 , because the appropriate Damköhler number to be used should be the ratio $\left(\delta_{\mathrm{F}} / \delta_{\mathrm{L}}\right)^{2}$, where $\delta_{\mathrm{F}}=\delta_{\mathrm{m}} / \beta$ is the characteristic size of the flame front edge. This is smaller than $\delta_{\mathrm{m}}$ by a factor $\beta$, typically of order 10 , equal to the effective non-dimensional overall activation energy of the reaction.

The ratio $U_{\mathrm{F}} / S_{\mathrm{L}}$ is also dependent on the thermal expansion, measured by the ratio, $T_{\mathrm{s}} / T_{0}$, between the stoichiometric flame temperature, and the initial fuel and air temperature. It is also dependent, although more weakly, on $S_{\mathrm{L}}$. Preliminary calculations of the function $U_{\mathrm{F}} /$ $S_{\mathrm{L}}=F\left(D_{a}\right)$, that we have carried out for undiluted methane/air flames, lead to the results that $U_{\mathrm{F}} / S_{\mathrm{L}}$ approaches a value close to 3 as the Damköhler number increases. In the absence of gravity forces, the flow velocity is $U$, in our case constant, and for small values of the lift-off height, when $\delta_{\mathrm{m}}^{2}=D_{\mathrm{F}} z_{\mathrm{L}} / U, z_{\mathrm{L}}$ is given by:

$$
U / S_{\mathrm{L}}=F\left(S_{\mathrm{L}}^{2} z_{\mathrm{L}} / U D_{\mathrm{F}}\right) \text {. }
$$

The lift-off height $z_{\mathrm{L}}$ grows with dilution of the fuel due to the effect that this dilution has in lowering $S_{\mathrm{L}}$ (the effects of the dilution on the thermal expansion ratio are weaker). For large values of the Damköhler number $S_{\mathrm{L}}^{2} z_{\mathrm{L}} / U D_{\mathrm{F}}, F$ becomes independent of $D_{a}$. However, this asymptotic value of $U_{\mathrm{F}} / S_{\mathrm{L}}$ will grow due to the interacting thermal expansion effects of the different elements of the annular flame front when, for large lift-off heights, the thickness of the mixing layer is comparable to the jet radius. The thermal expansion effects will further increase the flame front velocity above its asymptotic value for the planar mixing layer. The evolution with $S_{\mathrm{L}}$ of this lift-off height can no longer be estimated using (7), and must be obtained by solving the conservation equations with detailed kinetics and transport. Gravity effects influence the lift-off height since the value of $\delta_{\mathrm{m}}^{2} / D_{\mathrm{F}}$ to be used in (7) is no longer given by $z_{\mathrm{L}} / U$, because the velocity $u_{\mathrm{M}}$ in the methane stream grows with $z$ and, more importantly, because $U_{\mathrm{F}}$ must be balanced by the flow velocity in the stoichiometric surface, which also grows with $z$.

Based upon these arguments, it is clear that, if one can change (increase or decrease) the laminar flame speed of the corresponding stoichiometric mixture at the flame front, then we should see a corresponding (decrease or increase) in the liftoff height. With fuel dilution, $S_{\mathrm{L}}$ decreases significantly, and, thereby, to maintain the local front velocity, the lift-off height $z_{\mathrm{L}}$ must grow. Rather than changing all of the elementary rates in the mechanism, sensitivity analysis (see, for example [23]) reveals that the laminar flame speed of methane-air systems is particularly sensitive to the reactions $\mathrm{H}+\mathrm{O}_{2} \leftrightarrow \mathrm{O}+\mathrm{OH}$ and $\mathrm{H}+\mathrm{CH}_{3}+$ $(\mathrm{M}) \leftrightarrow \mathrm{CH}_{4}(+\mathrm{M})$. We changed the $\mathrm{H}+\mathrm{O}_{2}$ reaction rate in the mechanism [2] to match the rate in

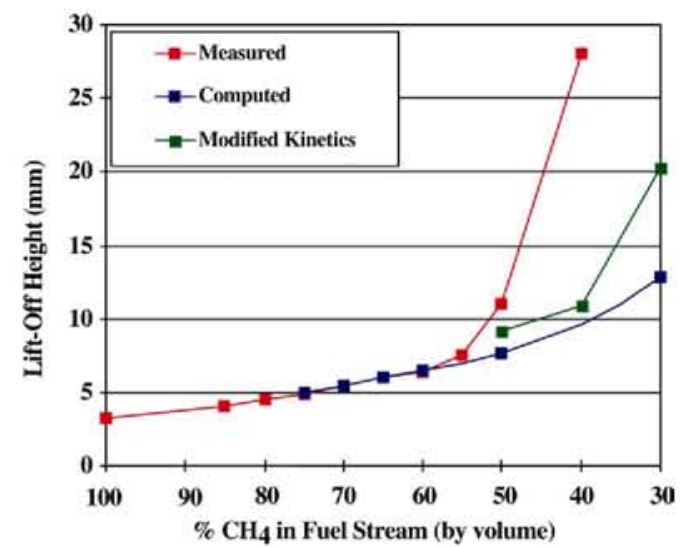

Fig. 5. Lift-off heights as a function of dilution level from measurement (red), computation (blue), and computation with slightly modified kinetics (green) for the normal gravity flame. (For interpretation of the references to color in this figure legend, the reader is referred to the web version of this paper.)

GRI-Mech 2.11, and the reaction rate for the $\mathrm{H}+\mathrm{CH}_{3}+(\mathrm{M})$ was arbitrarily increased by $10 \%$. Clearly, other rates could be modified, and a more systematic study/evaluation could be carried out to determine the critical rate(s) affecting the lift-off heights of diluted mixtures. However, these changes should be viewed as a first step to determine if, by varying $S_{\mathrm{L}}$, we can predict liftoff heights more in line with the experiments.

The two rate changes resulted in at most a 5$10 \%$ lowering of the computed laminar flame speed at the flame front for each of the diluted flames studied. Although these rate modifications made only minor adjustments to the lift-off heights of the $75 / 25$ and $65 / 35$ flames at normal gravity, they affected the more highly dilute mixtures more dramatically. In particular, lift-off heights for normal gravity flames $(50 / 50,40 / 60$, and $30 / 70$ ) increased by as much as $65 \%$ and are now closer to the experimental values (see Fig. 5). The results indicate that the lift-off heights for very dilute mixtures are clearly sensitive to the corresponding laminar flame speed and, in addition, kinetic mechanisms that work well at low to moderate dilutions may require refinement before they can be applied to cooler, more dilute methane-air diffusion flames near extinction.

\section{Summary}

We have imaged excited-state $\mathrm{CH}$ (denoted $\mathrm{CH}^{*}$ ), and excited-state $\mathrm{OH}\left(\right.$ denoted $\left.\mathrm{OH}^{*}\right)$ in coflow laminar diffusion flames. The measurements were made both in normal gravity and on the NASA KC-135 reduced-gravity aircraft. 
We compared measurements and computations over a wide range of dilution levels. Our results indicate that the lift-off heights and flame shapes predicted by the computations are in excellent agreement with measurement for both normal gravity and reduced gravity flames at low dilution levels but, as the fuel mixture is increasingly diluted, the lift-off heights become underpredicted. This trend continues until the computations predict stable flames at highly dilute fuel mixtures beyond the $1 g$ experimental blow-off limit. Asymptotic analysis has indicated the importance of the laminar flame speed of the fuel-air mixture at the stoichiometric flame front in controlling the liftoff height. By adjusting the rates of two key reactions in the methane-air mechanism, we have been able to more closely match the experimental lift-off values at normal gravity. The work indicates that reaction sets that work well at low to moderate dilutions may require refinement before they can be applied to cooler, more dilute methane-air diffusion flames near extinction.

\section{Acknowledgments}

The support of NASA under Grant NAG31939 is gratefully acknowledged. NASA Glenn personnel were extremely helpful from the early planning stages to the final nuts and bolts of flying the experiment successfully. In particular, we thank Jack Kolis, Eric Neumann, Karen Weiland, Joe Wilson, Jim Withrow, and John Yaniec.

\section{References}

[1] M.D. Smooke, P. Lin, J. Lam, M.B. Long, Proc. Combust. Inst. 23 (1991) 575.

[2] M.D. Smooke, Y. Xu, R.M. Zurn, P. Lin, J.H. Frank, M.B. Long, Proc. Combust. Inst. 24 (1992) 813.

\section{Comments}

Kalyan Annamalai, Texas A\&M University, USA. One way to determine blow off is to look at the velocity, $V_{x}$, along the stoichiometric contour and if the velocity at the flame tip exceeds laminar burning velocity, then blow off is likely to occur. Have you determined blow off conditions for your experiment and how does your model compare with data? What about the velocity, $V_{x}$, along the stoichiometric contour?

Reply. Blow-off conditions were determined experimentally but, for these limiting cases, the computational model predicted a stable flame. The fact that the computational model was unable to predict the blow-off conditions was a key result of the paper. While we have done
[3] M.D. Smooke, A. Ern, M.A. Tanoff, B.A. Valdati R.K. Mohammed, D.F. Marran, M.B. Long, Proc. Combust. Inst. 26 (1996) 2161.

[4] D.F. Marran, Quantitative Two-Dimensional Laser Diagnostics in Idealized and Practical Combustion Systems, $\mathrm{PhD}$ thesis, Yale University, New Haven, CT, 1996.

[5] K.T. Walsh, M.B. Long, M.A. Tanoff, M.D. Smooke, Proc. Combus. Inst. 27 (1998) 615.

[6] C.T. Bowman, R.K. Hanson, D.F. Davidson, W.C. Gardiner Jr, V. Lissianski, G.P. Smith, D.M. Golden, M. Frenklach, H. Wang, M. Goldenberg, GRI-Mech version 2.11, 1995. Available from http://www.gri.org.

[7] J. Luque, D.R. Crosley, Appl. Phys. B 63 (1996) 91.

[8] H.M. Hertz, G.W. Faris, Opt. Lett. 13 (1988) 351.

[9] J. Luque, J.B. Jeffries, G.P. Smith, D.R. Crosley, K.T. Walsh, M.B. Long, M.D. Smooke, Combust. Flame 122 (1-2) (2000) 172.

[10] C.J. Dasch, Appl. Opt. 31 (1994) 1146.

[11] K.T. Walsh, J. Fielding, M.B. Long, Opt. Lett. 25 (2000) 457

[12] J. Luque, D.R. Crosley, LIFBase Database and Spectral Simulation Program (Version 1.0), SRI International, Report MP 96-001 (1996).

[13] K. T. Walsh, J. Fielding, M.D. Smooke, M.B. Long, Proc. Combust. Inst. 28 (2000) 1973.

[14] J.B. Buckmaster, Prog. Energy Combust. Sci. 28 (2002) 435

[15] S. Ghosal, L. Vervisch, J. Fluid Mech. 415 (2000) 227.

[16] B.J. Lee, S.H. Chung, Combust. Flame 109 (1997) 163.

[17] P.N. Kioni, K.N.C. Bray, D.A. Greenhalgh, B. Rogg, Combust. Flame 116 (1999) 162.

[18] S. Ghosal, L. Vervisch, Combust. Flame 123 (2001) 646.

[19] T. Plessing, P. Terhoeven, N. Peters, M.S. Mansour, Combust. Flame 115 (1998) 335.

[20] Y.S. Ko, S.H. Chung, Combust. Flame 118 (1999) 151.

[21] G.R. Ruetsch, L. Vervisch, A. Liñán, Phys. Fluids 7 (6) (1995) 1070

[22] S.H. Chung, B.J. Lee, Combust. Flame 86 (1991) 62.

[23] M.D. Smooke (Ed.), Reduced Kinetic Mechanisms and Asymptotic Approximations for Methane-Air Flames. Springer-Verlag, Heidelberg, 1991.

extensive scalar measurements in these flames, velocities near the blow-off condition have not been measured.

V.R Katta, Innovative Scientific Solutions, USA. Wo presented a paper on flame stability and lift-off height [1]. We used different chemical mechanisms, including yours and the GRI, for the prediction of lift-off heights in methane air diffusion flames. We found that none of the mechanisms gave good predictions. Just the way you did, we performed a sensitivity analysis and found that $\mathrm{H}+\mathrm{CH}_{3}=\mathrm{CH}_{4}$ reaction is important in determining flame lift-off. We modified that in GRI mechanism 
and got excellent predictions. Did you change this reaction in GRI mechanism and try to predict flame lift-off heights for different dilutions. Maybe, you'll get good predictions for the lean flame also?

\section{Reference}

[1] F. Takahashi, V.R. Katta, Proc. Combust. Inst. 28 (2000) 2071-2078.

Reply. We have found that the mechanism we used in the paper gave superior lift-off agreement compared to GRI-Mech 2.11 (which produced lower lift-off heights) for flames that contained between 65 and $100 \%$ methane for both the ground-based and micro-gravity studies (Figs. 3 and 4). We cannot explain why, in your earlier studies, "none of the mechanisms gave good predictions." This is clearly contrary to our experience. However, as the level of nitrogen increases in the fuel jet, the level of agreement between our computational and experimental lift-off heights begins to deteriorate.

As indicated in the text, we changed the rate of $\mathrm{CH}_{3}+\mathrm{H}+(\mathrm{M})=\mathrm{CH}_{4}+(\mathrm{M})$ in our mechanism-not in GRI-Mech 2.11. While changing this rate in GRIMech 2.11 could tend to increase the lift-off levels of the lower dilution flames (which were smaller than those produced with our mechanism), it should be pointed out that the lift-off height of these flames did not change significantly when this rate was changed in our mechanism. As a result, it is likely that the lift-off heights of the higher dilution flames would not increase as much compared to our mechanism.

Jay Jeffries, Stanford University, USA. Results from the Yale chemical mechanism and from GRI Mech 2.11 were presented. Have you looked at the results predicted using GRI Mech. 3.0? What experimental targets would you recommend to tune the model?
Reply. We have not performed computations with GRI Mech 3.0. However, to tune the chemical mechanism, I would include quantities such as counterflow diffusion flame extinction strain rates as well as laminar flame speeds with high levels of dilution. The ability to predict accurately these quantities (as discussed in the paper) is critical if one wants to be able to compute accurate lift-off heights for highly diluted flames.

Bassam Dally, Adelaide University, Australia. Do you think that more detailed structure of the flame needs to be measured before you can conclude that the chemical kinetics needs refining? Can the lift off height, which is very sensitive to temperature, be a factor?

Reply. We have made a significant number of experimental and numerical comparisons of both temperature, major and minor species in coflow, laminar, methane-air diffusion flames. Various mechanisms have been used in these computations and we have developed a detailed understanding of why results closer to the experimental measurements are produced with one mechanism as opposed to another. As was discussed in the paper, various numerical (error tolerances, grid resolution) and modeling (variation of transport coefficients, modification of far field and down stream boundary conditions) parameters were varied to determine if these contributed to the lift-off height discrepancy. All produced minor variations that indicated that chemistry was the major factor. As is illustrated in the paper, small changes to two key reactions in the mechanism produced dramatic variations in the lift-off height. The explanation for this behavior is contained in the asymptotic analysis discussion. The results are clear that kinetic mechanisms that work well at low to moderate dilutions may require refinement before they can be applied to cooler, more dilute methane-air diffusion flames near extinction. 